\title{
King or royal family? Testing for species boundaries in the King Cobra, Ophiophagus hannah (Cantor, 1836), using morphology and multilocus DNA analyses
}

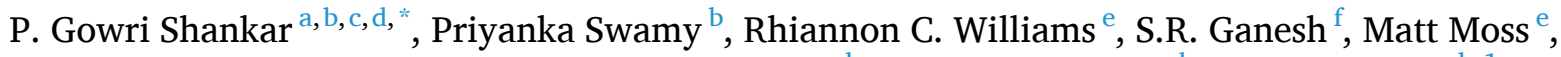 \\ Jacob Höglund $^{c}$, Indraneil Das ${ }^{g}$, Gunanidhi Sahoo ${ }^{\text {h }}$, S.P. Vijayakumar ${ }^{\mathrm{d}}$, Kartik Shanker ${ }^{\mathrm{b}, 1}$, \\ Wolfgang Wüster ${ }^{e, 1}$, Sushil K. Dutta ${ }^{a, i, 1}$ \\ ${ }^{a}$ Maharaja Shrirama Chandra Bhanja Deo University, Baripada, Takatpur, Odisha, India \\ ${ }^{\mathrm{b}}$ CES, Indian Institute of Science, Bangalore, India \\ ${ }^{\mathrm{c}}$ Department of Ecology and Genetics, Evolutionary Biology Centre, Uppsala University, Sweden \\ ${ }^{\mathrm{d}}$ Kālinga Foundation, Agumbe, Karnataka, India \\ ${ }^{\mathrm{e}}$ Molecular Ecology and Evolution at Bangor, School of Natural Sciences, Bangor University, Environment Centre Wales, Bangor LL57 2UW, Wales, UK \\ ${ }^{\mathrm{f}}$ Chennai Snake Park, Raj Bhavan Post, Chennai, India \\ ${ }^{\mathrm{g}}$ Institute of Biodiversity and Environmental Conservation, Universiti Malaysia Sarawak, 94300 Kota Samarahan, Malaysia \\ ${ }^{\mathrm{h}}$ Department of Zoology, Utkal University, Bhubaneswar, India \\ ${ }^{i}$ Department of Zoology, Assam Don Bosco University, Tapesia, Assam, India
}

\section{A R T I C L E I N F O}

\section{Keywords:}

Species delimitation

King cobra complex

Endemic

Western Ghats

Phylogenetics

Phylogeography

\begin{abstract}
A B S T R A C T
In widespread species, the diverse ecological conditions in which the populations occur, and the presence of many potential geographical barriers through their range are expected to have created ample opportunities for the evolution of distinct, often cryptic lineages. In this work, we tested for species boundaries in one such widespread species, the king cobra, Ophiophagus hannah (Cantor, 1836), a largely tropical elapid snake distributed across the Oriental realm. Based on extensive geographical sampling across most of the range of the species, we initially tested for candidate species (CS) using Maximum-Likelihood analysis of mitochondrial genes. We then tested the resulting CS using both morphological data and sequences of three single-copy nuclear genes. We used snapclust to determine the optimal number of clusters in the nuclear dataset, and Bayesian Phylogenetics and Phylogeography (BPP) to test for likely species status. We used non-metric multidimensional scaling (nMDS) analysis for discerning morphological separation. We recovered four independently evolving, geographically separated lineages that we consider Confirmed Candidate Species: (1) Western Ghats lineage; (2) Indo-Chinese lineage (3) Indo-Malayan lineage; (4) Luzon Island lineage, in the Philippine Archipelago. We discuss patterns of lineage divergence, particularly in the context of low morphological divergence, and the conservation implications of recognizing several endemic king cobra lineages.
\end{abstract}

\section{Introduction}

Species that were long thought to be 'widespread' and 'variable', when systematically studied, have frequently turned out to contain previously unrecognized diversity, and to comprise species complexes, typically consisting of multiple allopatric species, each inhabiting a smaller geographic area (e.g., Welton et al., 2010, 2014; Siriwut et al.,2015; Wüster et al., 2018). However, testing for species boundaries can be complicated in cryptic species that are superficially indistinguishable due to strong resemblance in morphology, behavior and ecology (Steyskal, 1972; Bickford et al., 2007). In recent years, molecular studies have improved the understanding of cryptic species across biogeographic regions (Pfenninger \& Schwenk, 2007; Vodă et al., 2014).

This issue is particularly acute in the Oriental tropics, a region with diverse geological histories and heterogenous contemporaneous climate and climax vegetation types (Archbold et al., 1982; Woodruff, 2010;

\footnotetext{
* Corresponding author at: Maharaja Shrirama Chandra Bhanja Deo University, Baripada, Takatpur, Odisha, India.

E-mail address: gowrishankar.pogiri@gmail.com (P. Gowri Shankar).

1 Joint last authors.
} 